\title{
Preditores da Intenção de Abandono Profissional em Caminhoneiros
}

\section{Predictors of Intention to Leave Profession in Truck Drivers}

\author{
Michelle Engers Taube (orcid.org/0000-0002-7865-953X)1 \\ Mary Sandra Carlotto (orcid.org/0000-0003-2336-5224)2
}

\begin{abstract}
Resumo
Apesar da importância da retenção de caminhoneiros, há pouca compreensão acerca das variáveis e suas interações para explicar a intenção do abandono profissional. O objetivo deste estudo foi avaliar as variáveis sociodemográficas, laborais, psicossociais, estressores ocupacionais e a satisfação no trabalho como possíveis preditores da intenção de abandono em uma amostra de 565 caminhoneiros brasileiros. Como instrumentos de avaliação, foram utilizadas as Subescalas de Tendência ao Abandono, Satisfação no Trabalho e Riscos Psicossociais, questionários de variáveis sociodemográficas, laborais e estressores ocupacionais. Os resultados obtidos por meio da análise de regressão linear múltipla apontaram um modelo explicativo para a tendência ao abandono composto por sete variáveis que conjuntamente explicaram $29,2 \%$ da variância, sendo a satisfação no trabalho a variável de maior poder preditivo $(13,2 \%)$. O estudo sugere ações de retenção desses profissionais aos gestores do transporte, com o objetivo de evitar a rotatividade dessa categoria.
\end{abstract}

Palavras-chave: Tendência ao abandono profissional. Caminhoneiros. Estressores ocupacionais. Riscos psicossociais.

\begin{abstract}
Despite the importance of truck driver's retention, there is not enough understanding about the variables that interact to explain the intention to leave the profession. The objective of this study was to evaluate variables such as, sociodemographic, labor, psychosocial, occupational stressors and satisfaction with work, as possible predictors of abandonment, in a sample of 565 Brazilian truck drivers. For data capture purposes, a set of assessment tools was used. It was composed by: Subscales of Tendency to Abandonment, Job Satisfaction and Psychosocial risks, sociodemographic, labor and occupational stressors questionnaire. The results obtained by multiple linear regression analysis, have indicated seven variables to explain the model variance of $29,2 \%$, with job satisfaction having the most significant prediction capacity $(13,2 \%)$. The study suggests some retention actions by the management level on truckload carriers, in order to avoid the turnover of this job category.
\end{abstract}

1 Universidade do Vale do Rio dos Sinos (Unisinos), São Leopoldo, Brasil. E-mail: michelletaube@hotmail.com.

${ }^{2}$ Universidade do Vale do Rio dos Sinos (Unisinos), São Leopoldo, Brasil. E-mail: mscarlotto@gmail.com. 
Keywords: Intention to leave profession. Truck drivers. Occupational stressors. Psychosocial risks. 


\section{Introdução}

O sistema de transporte de cargas é essencial para a movimentação da economia e suprimento de demanda no Brasil, sendo o motorista de caminhão agente fundamental desse sistema com expressiva presença no mercado de trabalho (Masson \& Monteiro, 2010). Segundo a Confederação Nacional do Transporte (CNT), em 2016, o Brasil contava com 2.645.992 motoristas de caminhão operando no território brasileiro, movimentando uma média de $61,1 \%$ da carga transportada no País. Embora o número seja expressivo, o deficit de caminhoneiros no Brasil atinge $13 \%$ da frota das empresas, ou seja, há uma carência de cerca de 100 mil motoristas (Associação Nacional de Transportes de Cargas e Logística, 2017).

Essa insuficiência pode ser resultado de uma mudança cultural (Movimento União Brasil Caminhoneiro, 2017), devido ao declínio da intenção desses profissionais em passar a profissão ao filho (Tolentino, 2012; Silva, 2015), pelo fato de os incentivos tradicionais não serem mais suficientes para fidelizar esse profissional (Swartz, Douglas, Roberts \& Overstreet, 2017) e pela própria natureza dessa ocupação, por ser um local de trabalho móvel e variado, facilitando assim a mudança de emprego (Suzuki, Crum \& Pautsch, 2009).

Pesquisas com caminhoneiros (CNT, 2016; Tolentino, 2012) referem que a maioria desses trabalhadores ingressa na profissão por ser um sonho de infância e de adolescência $(70 \%)$ - também é a maioria que manifesta interesse em abandonar a profissão (60\%) por considerá-la desgastante $(34,9 \%)$; de baixa qualidade de vida (55\%); pouca valorização profissional (11\%); pela necessidade de ausentarse por longos períodos de casa e da família, comprometendo o convívio familiar (32,1\%).

O conceito de intenção de abandono é definido como processo psicológico de natureza cognitiva e afetiva que se inicia com a análise da satisfação do trabalho, possibilidades de mercado, custos e benefícios da decisão de saída da profissão ou do local de trabalho (Mobley, 1977). A intenção de abandono sugere uma capacidade de predição do rompimento final do vínculo, indicando ser mais reveladora do que o desligamento definitivo (Brewer, Kovner, Greene \& Cheng, 2009; Zhang \& Feng, 2011).

Delfgaauw (2007) refere que existe uma diferença entre a intenção de abandonar a profissão e a intenção de abandonar o empregador, sendo que a motivação para cada uma é diferente. $O$ autor sugere que a insatisfação com aspectos do trabalho da organização sugere abandono do empregador e com aspectos da função, o abandono é da profissão.

Especificamente, em caminhoneiros, a intenção de abandono da profissão pode estar relacionada ao estresse causado pela solidão, pelas restrições de participação da vida familiar, problemas relacionados à incerteza do apoio à saúde, a discriminação e o impacto estressante dos regulamentos governamentais (Williams, Thomas \& Liao-Troth, 2017). A idade e a experiência profissional também se apresentam como preditores da intenção ao abandono, na medida em que caminhoneiros com mais idade e mais experiência são menos propensos a abandonar o trabalho, em virtude da capacidade 
de avaliação de sua empregabilidade e de estar habituado ao rigor das condições de trabalho (Nadler \& Kros, 2014; Suzuki et al., 2009).

O conflito trabalho/família e a falta de autonomia no trabalho são considerados preditores da intenção de abandonar a profissão e o empregador (Lannoo \& Verhofstadt, 2016). Os autores discorrem que a falta de apoio no trabalho, a forma de comunicar as mudanças de agenda e um contrato temporário podem ser preditores do abandono do trabalho; e a falta de realização e um ambiente de trabalho exigente, preditores do abandono da profissão.

O custo da rotatividade tem preocupado empregadores devido à necessidade de treinamento e orientação (Nadler \& Kros, 2014), pois a substituição frequente desses profissionais acarreta muitas desvantagens para as empresas transportadoras, entre as quais: a perda de habilidades e experiência do caminhoneiro; a redução da qualidade do serviço ao cliente; o aumento dos custos operacionais; a insegurança rodoviária (Curtis \& Wright, 2001; Suzuki, 2007; Suzuki et al., 2009), além do risco frequente de acidente de trânsito (Staplin \& Gish, 2005).

As taxas elevadas de rotatividade geram sobrecarga aos motoristas ativos no trabalho, o que culmina em afastamentos devido ao excesso de demandas, consequência do deficit de quadro de pessoal (LeMay, Williams \& Garver, 2009). A rotatividade do motorista de caminhão afeta $\mathrm{o}$ desempenho da cadeia de suprimento, devido ao seu impacto na segurança da operação e na capacidade produtiva do serviço rodoviário (Cantor, Macdonald \& Crum, 2011).

Uma vez formada a intenção de abandono, altera-se a percepção do profissional acerca do seu trabalho e do ambiente em que atua (Macedo, 2008). De acordo com a autora, existem dois caminhos: o positivo, que é quando o profissional se dá conta dessa intenção e muda sua percepção; e o negativo, quando procura outros elementos negativos para embasar sua decisão, o que gera maior insatisfação e menor comprometimento.

A insatisfação no trabalho pode ser considerada um preditor do abandono, que, quando associada aos riscos psicossociais, é preditor, também do conflito trabalho/família e de papéis, aumentando as possibilidades de abandono (Hom \& Kinicki, 2001). A satisfação no trabalho apresenta um efeito mediador em relação aos estressores ocupacionais e à intenção de abandonar, sendo a carga de trabalho o estressor com maior predição sobre o abandono (Jou, Kuo \& Tang, 2013). As condições dos equipamentos e ferramentas de trabalho também estão vinculadas às intenções de abandono, uma vez que boas condições contribuem para $\mathrm{o}$ atendimento às demandas $\mathrm{e}$ ao comissionamento adequado (Min \& Lambert, 2002).

Nesse sentido, pesquisar e ampliar o conhecimento sobre os fatores associados à intenção do abandono é importante para subsidiar gestores na implantação de políticas de gestão que visem mudar as condições que fundamentam a decisão de permanecer na ou abandonar a profissão. Assim, o estudo de delineamento observacional, analítico e de corte transversal teve como objetivo avaliar as variáveis sociodemográficas, laborais, psicossociais, estressores ocupacionais e a satisfação com o trabalho como possíveis 
preditoras da intenção de abandono em uma amostra de 565 caminhoneiros.

\section{Método}

\section{Participantes}

A amostra não probabilística constituiuse de 565 motoristas de caminhão, brasileiros, em atividade laboral há mais de quatro meses tempo de impacto dos estressores sobre a saúde, de acordo com estudo longitudinal realizado por Schonfeld (1996). Foram utilizados como critérios de seleção: sexo masculino, devido ao predomínio de 99,8\% na profissão (CNT, 2016); ser portador de Carteira Nacional de Habilitação $(\mathrm{CNH})$ de categoria "C" ou "E". O tamanho da amostra seguiu as orientações de Field (2009), sendo o número de participantes igual a 50, adicionado a oito vezes o número de variáveis independentes, para a análise dos resultados e confiabilidade das informações. No caso do presente estudo, foram utilizados 35 preditores, assim a amostra mínima prevista foi de 330 participantes.

Entre os participantes do estudo, a idade média foi de 37,7 anos (DP $=9,44$, amplitude $=20$ a 71); com companheiro(a) fixo(a) $(86,2 \%, \mathrm{n}=487)$; filhos $(77,2 \%, \mathrm{n}=$ 436); escolaridade em nível de Ensino Médio $(52,9 \%, \mathrm{n}=299)$. Os salários médios eram de $\mathrm{R} \$ 2.808,38(\mathrm{DP}=1.105,22$, amplitude $=1.000$ a 15.000). Em relação às características laborais, o vínculo empregatício efetivo foi referido pela maioria $(85,8 \%, \mathrm{n}=485)$; viajavam sem acompanhante $(89,2 \%, \mathrm{n}=504)$; e tinham uma média de 11,7 anos de atuação como caminhoneiro (DP $=9,505$, amplitude $=1$ a 45).
Trabalhavam, em média, 24 dias ao mês (DP = 2,34, amplitude $=15$ a 30); a carga horária diária era, em média, de 12 horas $(\mathrm{DP}=2,41$, amplitude 5 a 18). Quanto ao tipo de rota, os caminhoneiros que viajavam pequenos percursos, rota curta, corresponderam a 54,2\% ( $\mathrm{n}=306$ ); os que ficavam de 2 a 6 dias em viagem/trabalho, rota média, $14,2 \%(\mathrm{n}=84)$; e os que viajavam por mais de 7 dias, que percorriam longos trechos rodoviários, rota longa, totalizaram 24,4\% ( $\mathrm{n}=138)$. Quanto ao tipo de carga, a maior parcela transportava madeira $(37,7 \%, \mathrm{n}=213)$ e cargas variadas $21,8 \%(123)$.

\section{Instrumentos}

Os dados foram coletados por meio dos seguintes instrumentos autoaplicáveis constituídos de quatro blocos:

1. Questionário sociodemográfico (idade, situação conjugal, filhos, escolaridade) e laboral (tipo de vínculo, presença de acompanhante nas viagens, quantidade de dias que se encontra em viagem/trabalho, quantidade de dias de folga, remuneração, carga horária trabalhada/dia, tempo de atuação profissional e tipo de carga transportada).

2. Subescalas de Tendência ao Abandono e Satisfação no Trabalho da Bateria de Avaliação de Riscos Psicossociais (Unidad de Investigación Psicosocial de la Conducta Organizacional - Unipsico) de Gil-Monte (2005), traduzida e 
adaptada para o presente estudo. A Subescala de Tendência ao Abandono (quatro itens, $\alpha=0,83$, ex.: Você pensa em mudar de profissão) também seguiu o mesmo critério avaliativo, de frequência de cinco pontos (0 "nunca" a 4 “diariamente”). A Subescala de Satisfação no Trabalho (seis itens, $\alpha$ $=0,76$, ex.: Qual é a sua satisfação em relação às oportunidades que $\mathrm{O}$ seu trabalho lhe oferece para fazer coisas de que gosta) foi avaliada por uma escala de cinco pontos (0 "muito insatisfeito" a 4 "muito satisfeito").

3. Subescalas da Escala de Estressores Psicossociais no Contexto Laboral (Ferreira et al., 2015). Foram utilizadas seis subescalas, totalizando 31 itens: 1. Conflito e ambiguidade de papéis (cinco itens, $\alpha=0,77$, ex.: Recebo instruções contraditórias sobre o que fazer no trabalho), referindo-se à falta de clareza em relação às próprias funções e ao recebimento de demandas contraditórias sobre as funções desempenhadas. 2. Sobrecarga de papéis (seis itens, $\alpha=0,71$, ex.: $\mathrm{O}$ meu trabalho tem um ritmo acelerado), que consiste no excesso de tarefas solicitadas ao empregado e que, às vezes, ele desempenha. 3. Falta de suporte social (seis itens, $\alpha=$ 0,77, ex.: Recebo ajuda dos meus superiores quando tenho algum problema pessoal), sendo as dificuldades advindas da falta de suporte emocional recebido pelos colegas e superiores, no cotidiano do trabalho. 4. Falta de autonomia (cinco itens, $\alpha=0,71$, ex.: Posso decidir quando tirar minhas férias), que se refere às dificuldades de planejar e de tomar decisões acerca das próprias tarefas, controles de trabalho. 5. Conflito trabalho/família (cinco itens, $\alpha=0,75$, ex.: Tenho dificuldade de conciliar as questões do trabalho com questões familiares), consiste em implicar uma relação de incompatibilidade entre as responsabilidades associadas à família e ao trabalho. 6. Pressão do grau de responsabilidade (quatro itens, $\alpha=$ 0,77, ex.: Os meus erros podem interferir negativamente na vida de outras pessoas), sobre o empregado no desempenho de suas funções, em relação a pessoas e equipamentos, item que também foi avaliado por meio de uma escala de frequência de cinco pontos (0 "nunca" a 4 "diariamente").

4. Questionário de estressores ocupacionais em caminhoneiros, elaborado especificamente para este estudo com base na literatura acerca dos estressores presentes nessa profissão (Cavagioni, Pierin, Batista, Bianchi \& Costa, 2009; Delfino \& Moraes, 2015; Marqueze, Ulhôa \& Moreno, 2012; Resende, Souza \& Cerqueira, 2010; Silva, Luz, 
Vasconcelos, Marqueze \& Moreno, 2016; Ulhôa et al., 2010; Van der Beek, 2012). Esse questionário contempla 14 itens que avaliaram a infraestrutura das rodovias e dos postos de paradas (condições de estradas, postos de atendimento, condições de realização dos repousos, local para alimentação); a insegurança (assaltos e roubos); o trânsito (congestionamento, acidentes); a poluição (sonora, ambiental); a jornada de trabalho; a remuneração; os sistemas eletrônicos de controle; e as equipes de apoio operacional. Os itens foram avaliados por meio de uma escala de quatro pontos (1 "nada estressante" a 4 "muito estressante").

\section{Procedimentos de coleta de dados}

Para a coleta dos dados, primeiramente, foi realizado contato com os locais para a explanação dos objetivos e para o estabelecimento da logística da coleta de dados, que foi realizada no período de março a junho de 2017. Foi efetivado um estudo piloto de instrumento no mês de fevereiro de 2017, com 12 caminhoneiros não pertencentes à amostra do estudo. Não foram detectadas dificuldades de compreensão das instruções e itens do instrumento, somente a sugestão de um estressor ocupacional foi indicado: equipe de apoio operacional, sendo este incorporado ao instrumento.
A aplicação foi realizada de forma coletiva e presencial pela pesquisadora e por profissionais psicólogas previamente treinadas e a coleta foi realizada em seis empresas do segmento de transporte, três Centros de Formação de Condutores (CFCs), dois postos de combustíveis e 15 turmas de aprendizagem (Curso de Movimentação de Produtos Perigosos e Cargas Indivisíveis) de cursos destinados a esses profissionais.

A coleta iniciou-se com o convite à participação e posterior leitura e assinatura do Termo de Consentimento Livre e Esclarecido; os instrumentos foram preenchidos nos próprios locais e recolhidos logo depois do término; o tempo de preenchimento foi de aproximadamente 50 minutos; sendo o estudo aprovado pelo Comitê de Ética em Pesquisa da Universidade do Vale do Rio dos Sinos (Unisinos), sob o número CAAE: 62418116.7 .0000 .5344$.

\section{Procedimentos de análise de dados}

Os dados foram analisados no programa Statistical Package for Social Sciences, versão 22 (PASW, Inc., Chicago, IL); estatísticas descritivas foram realizadas para calcular as frequências, médias e desvios-padrão; e a força e a direção das relações entre as variáveis foram determinadas usando a medida do coeficiente de correlação de Pearson.

Foi realizada a análise de regressão linear múltipla (método Stewise). Assumiu-se como variável dependente, a intenção de abandono profissional e como variáveis independentes, as sociodemográficas, as 
laborais, os estressores ocupacionais, os riscos psicossociais e a satisfação com o trabalho.

Foram testados os pressupostos de regressão linear múltipla sem identificação de violação que contraindicasse sua utilização, de acordo com os parâmetros de Field (2009). Identificou-se ausência de multicolinearidade entre as variáveis, pois todos os valores das correlações ficaram abaixo de 0,413 , o valor de Variance Inflation Factor (VIF) situou-se abaixo de $4(1,100)$ e o valor de tolerância foi inferior a 1 (0,909). A análise do coeficiente de DurbinWatson situou-se próximo a $2(1,810)$, indicando a independência da distribuição e a não correlação dos resíduos. A distância de Cook apresentou valor de 0,003 , inferior a 1 , revelando não existir preditores atípicos e um adequado ajuste do modelo.
A seleção das variáveis preditoras adotou o nível de significância de $\mathrm{p}<0,05$. $\mathrm{Na}$ análise de regressão, a magnitude do efeito foi obtida pelos coeficientes de regressão padronizados e calculados no modelo final, de acordo com Marôco (2007).

\section{Resultados}

Na Tabela 1, verifica-se que a média mais elevada refere-se aos estressores ocupacionais, assaltos e roubos; e a menor ao risco psicossocial, conflito e ambiguidade de papéis. Quanto aos alfas encontrados, estes variaram de bons índices de fidedignidade a aceitáveis (Marôco \& Garcia-Marques, 2006).

Tabela 1. Amplitude de resposta, alfa, média e desvio padrão dos instrumentos utilizados

\begin{tabular}{lcccc}
\hline & Escala & $\alpha$ & $\mathrm{M}$ & $\mathrm{DP}$ \\
\hline Tendência ao abandono & $0-4$ & 0,71 & 1,70 & 0,97 \\
Conflito e ambiguidade de papéis & $0-4$ & 0,67 & 0,99 & 0,73 \\
Sobrecarga de papéis & $0-4$ & 0,65 & 2,30 & 0,84 \\
Falta de suporte social & $0-4$ & 0,84 & 2,17 & 0,87 \\
Falta de autonomia & $0-4$ & 0,67 & 2,07 & 0,86 \\
Conflito trabalho/família & $0-4$ & 0,61 & 1,20 & 0,77 \\
Pressão do grau de responsabilidade & $0-4$ & 0,89 & 2,11 & 1,27 \\
Satisfação no trabalho & $0-4$ & 0,82 & 2,52 & 0,75 \\
Estressores ocupacionais & $1-4$ & - & 2,30 & 0,53 \\
Condições das estradas & - & - & 2,71 & 0,84 \\
Postos de atendimento & - & - & 1,89 & 0,82 \\
Condições de repouso & - & - & 1,67 & 0,87
\end{tabular}




$\begin{array}{lcccc}\text { Locais para alimentação } & - & - & 1,50 & 0,76 \\ \text { Assalto } & - & - & 2,98 & 1,05 \\ \text { Roubos } & - & - & 2,98 & 1,06 \\ \text { Congestionamentos } & - & - & 2,81 & 0,97 \\ \text { Acidentes } & - & - & 2,90 & 0,99 \\ \text { Poluição sonora } & - & - & 2,57 & 0,93 \\ \text { Poluição ambiental } & - & - & 2,81 & 0,96 \\ \text { Jornada de trabalho } & - & - & 1,81 & 0,88 \\ \text { Remuneração } & - & - & 1,95 & 0,99 \\ \text { Sistemas eletrônicos de controle } & - & - & 1,96 & 0,98 \\ \text { Equipes de apoio } & - & - & 1,69 & 0,87\end{array}$

Fonte: Elaborada pelas autoras.

A Tabela 2 apresenta os resultados da análise de regressão linear múltipla, que considerou a tendência ao abandono da profissão como variável dependente e, como variáveis preditoras, as sociodemográficas, laborais, estressores ocupacionais, satisfação no trabalho e riscos psicossociais. A análise dos preditores do abandono evidenciou um modelo explicativo constituído por sete variáveis, que, conjuntamente, explicaram $29,2 \%$ da variância, sendo a satisfação no trabalho a variável de maior poder explicativo $(13,2 \%)$.

O conjunto de variáveis que explicaram a tendência ao abandono indica que quanto maior for a satisfação no trabalho, menor a intenção de abandonar a profissão. Quanto maior os estressores jornada de trabalho e a remuneração, as horas trabalhadas no mês, os riscos psicossociais pressão do grau de responsabilidade e o conflito e ambiguidade de papéis e considerar a poluição ambiental como um estressor, maior a tendência ao abandono.

Os resultados revelam uma magnitude de efeito elevada $\left(R^{2}-0,132\right.$ a 0,292$)$, de acordo com os parâmetros recomendados por Marôco (2007). Nesse sentido, indica que as relações identificadas, possivelmente, também estarão presentes na população-alvo de caminhoneiros. 
Tabela 2. Modelo de análise de regressão linear múltipla da tendência ao abandono

\begin{tabular}{|c|c|c|c|c|c|c|c|c|}
\hline Variáveis & $\boldsymbol{R}$ & $R^{2}$ & $\boldsymbol{R}^{2}$ ajustado & B & SE & $\beta$ & $t$ & $p$ \\
\hline \multicolumn{9}{|l|}{ Tendência ao abandono } \\
\hline Satisfação no trabalho & 0,364 & 0,132 & 0,130 & $-0,260$ & 0,064 & $-0,203$ & $-4,038$ & $0,000 * *$ \\
\hline $\begin{array}{l}\text { Estressor jornada de } \\
\text { trabalho }\end{array}$ & 0,442 & 0,196 & 0,191 & 0,344 & 0,121 & 0,141 & 2,848 & $0,005^{* *}$ \\
\hline Estressor remuneração & 0,474 & 0,224 & 0,218 & 0,296 & 0,110 & 0,137 & 2,680 & $0,008^{* *}$ \\
\hline Carga horária/mês & 0,498 & 0,248 & 0,239 & 0,002 & 0,001 & 0,141 & 3,033 & $0,003 * *$ \\
\hline $\begin{array}{l}\text { Pressão do grau de } \\
\text { responsabilidade }\end{array}$ & 0,516 & 0,266 & 0,255 & 0,097 & 0,035 & 0,130 & 2,822 & $0,005^{* *}$ \\
\hline $\begin{array}{l}\text { Conflito e ambiguidade de } \\
\text { papéis }\end{array}$ & 0,530 & 0,281 & 0,269 & 0,175 & 0,066 & 0,131 & 2,635 & $0,009 * *$ \\
\hline $\begin{array}{l}\text { Estressor } \\
\text { ambiental }\end{array}$ & 0,541 & 0,292 & 0,278 & 0,219 & 0,091 & 0,109 & 2,396 & $0,017 *$ \\
\hline F Model & & & & & 766 & & & \\
\hline
\end{tabular}

$* p \ll 0,05,{ }^{* *} p \ll 0,01$.

Fonte: Elaborada pelas autoras.

\section{Discussão}

Este estudo objetivou avaliar os fatores sociodemográficos, laborais, riscos psicossociais, satisfação no trabalho e os estressores ocupacionais como variáveis preditoras da tendência de abandono da profissão. Os resultados identificaram modelos explicativos compostos pelas variáveis, satisfação no trabalho, os estressores ocupacionais; jornada de trabalho, remuneração e poluição ambiental, a característica laboral; quantidade de horas trabalhadas no mês e os riscos psicossociais; pressão do grau de responsabilidade e o conflito e ambiguidade de papéis.

A análise dos preditores do abandono revelou que quanto maior é a satisfação no trabalho, menor é a tendência ao abandono; quanto maior os estressores jornada de trabalho e a remuneração, as horas trabalhadas no mês, os riscos psicossociais pressão do grau de responsabilidade e o conflito e ambiguidade de papéis e considerar a poluição ambiental como um estressor, maior a tendência ao abandono.

A satisfação no trabalho, variável que obteve o maior poder preditivo em relação à intenção de abandono, confirma a literatura, no que diz respeito à sua associação a abandonar a profissão (Ferreira \& Siqueira, 2005; Gomide, 1999; Siqueira, 2001). No caso específico dos caminhoneiros, pode ser entendida a partir de estudos que referem que, apesar da diversidade de ambientes e de condições de trabalho, dos quais decorrem vários agravos à sua saúde, os caminhoneiros estão satisfeitos com o trabalho (Huang et al., 2016; Ribeiro, 2008; Simmonds, 
2015). De acordo com o estudo de Huang et al. (2016), estar insatisfeito no trabalho aumentaria em 1,25 vezes a chance de rotatividade.

É consenso na literatura que os caminhoneiros enfrentam uma infinidade de estressores no trabalho (Cavagioni et al., 2009; Delfino \& Moraes, 2015; Marqueze et al., 2012; Resende et al., 2010; Silva et al., 2016; Van der Beek, 2012; Ulhôa et al., 2010). Portanto, o estresse ocupacional pode ser considerado um preditor para o abandono da profissão (Shattell, Apostolopoulos, Sönmez \& Griffin, 2010). A jornada de trabalho é o estressor mais citado nos estudos com esses profissionais (Apostolopoulos, Peachey \& Sönmez, 2011; Apostolopoulos, Sönmez, Shattell, Gonzales \& Fehrenbacher, 2013; Narciso \& Mello, 2017; Ulhôa et al., 2010), sendo atualmente a variável mais estudada e indicada como preditora de transtornos mentais comuns (Ulhôa et al., 2010).

A jornada de trabalho gera aumento da fadiga e do risco de acidente de trânsito, que dobra a partir das 12 horas de trabalho, bem como aumenta a partir de 5 horas e 30 minutos ininterruptas sem descanso (Narciso \& Mello, 2017). As longas jornadas de trabalho permitem inferir um repertório de riscos à atividade laboral desses profissionais, o que contribui para a tendência ao abandono da profissão (Apostolopoulos, Sönmez, Hege \& Lemke, 2016).

O número exagerado de horas de trabalho dos profissionais do transporte tornou esse segmento altamente competitivo, gerando pressões intensas sobre os motoristas, em relação a prazos de entrega e ao trabalho excessivo (Apostolopoulos et al., 2016). O pagamento por comissão instiga o trabalhador a produzir mais sem ser remunerado por isso (Oliveira, 2012; Simmonds, 2015) e essa condição incentiva longas jornadas e a falta de descanso adequado (Quinlan \& Wright, 2008). A percepção de rodar menos quilômetros em comparação aos demais colegas e observar discrepâncias entre a rodagem paga e a realizada associaram-se à intenção de abandono da profissão, conforme evidencia o estudo de Suzuki et al. (2009).

Esses resultados podem estar relacionados ao poder preditivo da remuneração em relação à intenção de abandono. $\mathrm{O}$ trabalho do caminhoneiro é baseado em rodagem e comissão, o que motiva a redução do tempo de descanso e, por consequência, pressão por entregas rápidas e por mais responsabilidades. Assim, a remuneração torna-se um estressor, devido a todas as consequências que ela gera, exigindo uma jornada exaustiva de produtividade, sem considerar as condições de segurança do trabalhador, favorecendo o desgaste e, como desfecho, a intenção de abandono. Um estudo evidenciou que uma remuneração total maior implica menores intenções de abandono (Suzuki et al., 2009); porém, em contrapartida, um maior comprometimento da saúde ocupacional desses trabalhadores (Apostolopoulos et al., 2016; Krueger, 2013).

Quanto à quantidade de horas trabalhadas no mês, pode-se pensar que, no caso do caminhoneiro, trabalhar uma grande quantidade de horas ao mês dispende um esforço adicional do profissional no trânsito, o que implica na responsabilidade por sua 
segurança viária e pela de outros condutores, além das exigências laborais, como entrega da mercadoria e cuidado com o caminhão. Em consequência, o excesso de horas trabalhadas resulta em redução de descanso e de horas de sono, fatores que comprometem $\mathrm{O}$ desenvolvimento da atividade do caminhoneiro.

É consenso na literatura que a exacerbada quantidade de horas de trabalho nessa profissão compromete as necessidades básicas do profissional, gerando fadiga, cansaço, maior propensão a acidentes e, concomitantemente, uso de substâncias estimulantes para manter-se acordado (Cavagioni et al., 2009; Delfino \& Moraes, 2015; Narciso \& Mello, 2017; Shattell et al., 2010; Van der Beek, 2012).

Os dias em viagem prevalecem em relação ao convívio com a família, ao lazer e ao tempo livre; o acesso à educação, aos serviços de saúde e às possibilidades de lazer é restrito nas rodovias (Apostolopoulos et al., 2016), o que adia a procura por atendimento médico nessa categoria de trabalhadores (Delfino \& Moraes, 2015). Estudos indicam correlação positiva entre quantidade de horas de trabalho e o conflito trabalho/família; ou seja, quanto mais horas são dispendidas com o trabalho, maior é o conflito (DiRenzo, Greenhaus \& Weer, 2011; Pinto, 2012; Silva \& Silva, 2015).

Resultado contrário ao obtido neste estudo, com relação à quantidade de horas de trabalho, foi encontrado nos estudos de Suzuki et al. (2009) e Nadler e Kros (2014). Nos estudos desses autores, conduzir menos indicava maior propensão ao abandono, porém, a remuneração se mantinha a mesma, situação não condizente com os caminhoneiros brasileiros, uma vez que a remuneração é composta por comissionamento de acordo com a rodagem.

As variadas demandas do caminhoneiro explicam o conflito e a ambiguidade de papéis. A profissão que tinha como principal atividade conduzir o caminhão passa a responsabilizar-se por uma variabilidade de demandas, como representar a empresa perante o cliente, atender às exigências de controladores e de rastreadores (Van der Beek, 2012), atuar no manejo da carga, conferência, amarração, acompanhamento e descarga, além da manutenção do veículo (Delfino \& Moraes, 2015).

A variabilidade das atividades presentes no exercício da profissão, somadas ao risco psicossocial, pressão do grau de responsabilidade, constitui o repertório de exigências que o caminhoneiro é obrigado a observar. A pressão do tempo gera risco, pois é exigida a entrega do produto no dia programado; porém, muitas vezes, esse tempo é insuficiente, uma vez que o profissional pode ser exposto a diversos agentes sobre os quais ele não tem controle, como as condições climáticas e rodoviárias (Shattell et al., 2010).

A responsabilidade também é estendida aos cuidados com o patrimônio. Tendo que lidar com o trânsito, o caminhoneiro fica mais vulnerável a acidentes devido a sua exposição diária e maior probabilidade de tempo em permanência nesse ambiente. Relacionado a esse contexto, as condições dos veículos ocupam o segundo lugar de importância para os caminhoneiros em termos de intenção de abandono (Min \& Lambert, 2002), pois dependem dessa condição a direção adequada e 
a composição salarial.

O estressor poluição ambiental ser considerado um preditor da intenção do abandono é um resultado recente na literatura (Shattell et al., 2010; Suzuki et al., 2009; Van der Beek, 2012), talvez por causa das implicações e divulgações dos efeitos ambientais e impactos na qualidade de vida. Esse resultado pode ser explicado considerando a exposição a diversos riscos relacionados ao ambiente, no exercício da profissão.

Também deve ser considerado o risco de derramamento ou explosão da carga que está sendo trasportada, devido ao tipo de produto que a compõem, às vezes causando grandes catástrofes ambientais. Alguns cursos são necessários para o transporte desses produtos (Movimentação de produtos perigosos - Mopp) devido à existência de riscos e alguns cuidados e treinamentos obrigatórios, quando necessário for, no sentido de contenção e emergência.

Diversos são os perigos presentes na condução de um veículo: a vibração do corpo, o ruído, os fatores climáticos, escapes de diesel e monóxido de carbono (Bigelow et al., 2012; Shattell et al., 2010; Van der Beek, 2012). A exposição excessiva pode causar comprometimento físico, como dores, alterações degenerativas da coluna vertebral e a perda da audição (Bovenzi \& Hulshof, 1999; Lings \& Leboeuf-Yde, 2000; Seshagiri, 1998). Esses riscos geram estresse na condução, frustração e exigência de um repertório de estratégias para lidar com esse estressor (Shattell et al., 2010). O abandono do trabalho, inicialmente, é temporário, via licença médica, para posterior afastamento previdenciário, sendo que essa interrupção do trabalho refere-se principalmente a desconfortos físicos desencadeados no exercício profissional (Delfino \& Moraes, 2015).

A rotatividade de caminhoneiros não impacta somente as empresas transportadoras, estendendo-se aos demais colegas de trabalho, que devem atender normalmente às demandas, gerando aumento na carga de trabalho e queda no desempenho (Mobley, 1982), com consequências no trânsito e na segurança da população (Delfino \& Moraes, 2015; Narciso \& Mello, 2017).

A redução das taxas de rotatividade de caminhoneiros é tema de muitos estudos, devido à sua magnitude, impactos na segurança viária e custos operacionais no segmento do transporte. Caminhoneiros experientes e produtivos têm buscado empregos fora do setor de transporte rodoviário (Jones, 2013), repercutindo em um aumento no número de acidente de trânsito, maiores despesas para as organizações em recrutamento e seleção, treinamento e redução de produtividade. Recomendam-se estratégias de retenção e de atração de novos profissionais e ações para aumentar a eficiência logística no sentido de diminuir os impactos negativos associados à falta de motoristas (Jones, 2013).

Este estudo visa a colaborar na construção de um modelo constituído por um conjunto de variáveis que predizem a intenção de abandono profissional e o planejamento e manutenção de ações de retenção dessa classe de trabalhadores, sugerindo subsídios para a construção de modelos de gerenciamento da rotatividade. Concentrar ações que visem à satisfação desses profissionais pode contribuir com a redução da rotatividade, bem como 
amenizar os riscos psicossociais e estabelecer uma jornada adequada de trabalho, que considere as condições desses trabalhadores e suas necessidades. Ações ambientais buscando uma melhor adaptação dos caminhoneiros, como a possibilidade de dirigibilidade em equipamentos que visem ao conforto ergonômico, térmico, físico e químico também podem contribuir para reduzir a intenção de abandono e as taxas de afastamento temporário.

\section{Forças, limitações do estudo e implicações para a prática}

O presente estudo, ao identificar os preditores da tendência de abandono em caminhoneiros, contribui para a construção de um modelo teórico sobre essa temática e ampliação do conhecimento da ciência psicológica a ser transferido para empresas, gestores e profissionais do setor rodoviário, desse importante fenômeno que gera repercussões no trânsito e na cadeia produtiva do transporte. Destacam-se como forças do presente estudo o uso de medidas internacionalmente aceitas e adaptadas para o Brasil e o elevado índice da magnitude do efeito de seus resultados, o que indica que as relações identificadas possivelmente também estarão presentes na população-alvo.

O estudo, todavia, apresenta algumas limitações, que devem ser consideradas na leitura dos resultados: a primeira diz respeito ao delineamento transversal, que impossibilita estabelecer relações de causalidade; a segunda é a utilização de medidas de autorrelato, que podem gerar um viés de resposta relacionado à desejabilidade social - especificamente, neste estudo, podem-se citar as questões relacionadas à satisfação no trabalho e à tendência ao abandono. Pode ser difícil para o caminhoneiro assumir que não está satisfeito na sua profissão e que deseja abandoná-la, pelo fato de, historicamente, a profissão ser marcada por desejos de novidades, de autonomia nas decisões, de liberdade, e de ganhos financeiros sem necessidade de elevada escolaridade; e a terceira refere-se ao fato de que parte da coleta foi realizada em empresas de trabalho dos participantes e em Centros de Formação de Condutores, que podem ter gerado viés de resposta, na medida em que o trabalhador pode ter tido algum desconforto em responder itens que podem comprometer sua permanência no emprego ou renovação de sua habilitação.

Os resultados obtidos sugerem a necessidade de aprofundamento por meio de estudos longitudinais mistos de múltiplas fontes, no sentido de ampliar a compreensão dos padrões comportamentais e na construção de modelos explicativos e preditivos de variáveis no campo da PSO (Abbad \& Carlotto, 2016). Tal delineamento permitiria ampliar o conhecimento sobre como se desenvolve, ao longo do tempo, o processo de intenção/abandono profissional e o acompanhamento do modelo e do poder preditivo identificado. Igualmente importante é a realização de investigações com amostras estratificadas por região, buscando analisar a influência de aspectos culturais e regionais no contexto laboral desses profissionais, bem como os estressores ocupacionais presentes em cada região, a fim de conhecer elementos que 
predizem o processo de intenção/abandono profissional.

Em relação às implicações para a prática, sugerem-se ações de retenção e de atração profissional, com base nos resultados apontados no estudo. A construção de um modelo de gestão baseado em ações a partir do monitoramento constante da satisfação no trabalho e estressores ocupacionais é uma medida que pode prevenir o abandono.

Sugerem-se ações voltadas à manutenção e conservação dos caminhões, visando minimizar o desconforto físico, pois os sistemas de ar-condicionado acoplado reduzem o impacto do ruído do motor e do tráfego, bem como a exposição durante a condução de escape de óleo diesel, devido à vedação da cabine. Locais adequados onde o motorista possa aguardar o carregamento e descarregamento da carga, também, pode ser uma medida cabível de proteção à exposição dos riscos físicos, químicos e climáticos.

Outra ação possível é a redução da jornada de trabalho, visando assegurar uma melhor qualidade de vida a esses profissionais, bem como uma redução das possibilidades de abandono. Um fator que chamou a atenção nesta pesquisa foi verificar que maior remuneração também prediz a intenção de abandono, favorecendo, assim, a redução da jornada de trabalho, devido à remuneração ser composta de comissão por rodagem. Uma equipe de apoio operacional capaz de contribuir, orientar e auxiliar os caminhoneiros nas atividades administrativas de sua responsabilidade pode auxiliar na minimização do risco de conflito e ambiguidade de papéis.
Ações observando essas diretrizes podem contribuir no sentido de evitar a intenção e, consequentemente, o abandono definitivo.

\section{Referências}

Abbad, G. S., \& Carlotto, M. S. (2016).

Analyzing Challenges Associated with the Adoption of Longitudinal Studies in

Work and Organizational Psychology.

Revista Psicologia Organizaçoes e Trabalho, 16(4), 340-348. Doi:

10.17652/rpot/2016.4.12585.

Apostolopoulos, Y., Sönmez, S., Hege, A., \& Lemke, M., (2016). Work Strain, Social Isolation and Mental Health of LongHaul Truckers. Occupational. Therapy in Mental Health, 32(1), 50-69.

Doi:10.1080/0164212X.2015.1093995

Apostolopoulos, Y., Sönmez, S., Shattell, M. M., Gonzales, C., \& Fehrenbacher, C. (2013). Health Survey of US Long-Haul Truck Drivers: Work Environment, Physical Health, and Healthcare Access. Work: $A$ Journal of Prevention, Assessment and Rebabilitation, 46, 113-123.

Apostolopoulos, Y., Peachey, A. A., \& Sönmez, S. (2011). The Psychosocial Environment of Commercial Driving: Morbidities, Hazards, and Productivity of Truck and Bus Drivers. In J. Langan-Fox \& C. L. Cooper (Eds.). Handbook of Stress in the Occupations (pp. 431-447). Northampton, USA: Edward Elgar. 
Associação Nacional do Transporte de Cargas e Logística (2017). Falta de caminhoneiros.

Recuperado de http://www.portalntc.org.br.

Bigelow, P. L., Betts, D., Hogg-Johnson, S., Anick, B. C., Sieber, W. K., Skinner, M., \& Jakubicek, P. (2012). Health, Safety, and Wellness of Truck Drivers in Canada. In Freight Demand Modeling: Tools for PublicSector Decision Making, Summary of a Conference (Vol. 40). Baltimore, Maryland: Transportation Research Board.

Bovenzi, M., \& Hulshof, C. T. J. (1999). An Updated Review of Epidemiologic Studies on the Relationship between Exposure to Whole-Body Vibration and Low Back Pain (1986-1997). International Arcbives of Occupational and Environmental Health, 72(6), 351-365.

Brewer, C. S., Kovner, C. T., Greene, W., \& Cheng, Y. (2009). Predictors of RNs' Intent to Work and Work Decisions 1 Year Later in a US National Sample. International Journal of Nursing Studies, 46(7), 940-956.

Cantor, D. E., Macdonald, J. R., \& Crum, M. R. (2011). The Influence of Workplace Justice Perceptions on Commercial Driver Turnover Intentions. Journal of Business Logistics, 32(3), 274-286.

Cavagioni, L. C., Pierin, A. M. G., Batista, K. D. M., Bianchi, E. R. F., \& Costa, A. L. S. (2009). Health Problems, Hypertension and Predisposition to Stress in Truck
Drivers. Revista da Escola de Enfermagem da USP, 43(SPE2), 1267-1271.

Doi:10.1590/S0080-62342009000600021.

Confederação Nacional do Transporte (CNT). (2016). Boletim Estatístico do Transporte.

Recuperado de http://www.cnt.org.br/Boletim/boletimestatistico-cnt.

Curtis, S., \& Wright, D. (2001). Retaining Employees - The Fast Track to Commitment. Management Research News, 24(8/9), 56-60.

Doi:10.1108/01409170110782964.

Delfgaauw, J. (2007). The Effect of Job Satisfaction on Job Search: Not Just Whether, but Also Where. Labour Economics, 14(3), 299-317.

Delfino, L. G., \& Moraes, T. D. (2015). Percepções sobre adoecimento para caminhoneiros afastados pelo sistema de previdência social. Estudos Interdisciplinares em Psicologia, 6(2), 113-137. Doi:10.5433/22366407.2015v6n2p113.

Dickter, D. N., Roznowski, M., \& Harrison, D. A. (1996). Temporal Tempering: An Event History Analysis of the Process of Voluntary Turnover. Journal of Applied Psychology, 86(3), 493-522.

DiRenzo, M., Greenhaus, J., \& Weer, C. (2011). Job Level, Demands, and Resources as Antecedents of Work-Family Conflict. Journal of Vocational Behavior, 78, 305-314. 
Ferreira, M. C., Milfont, T. L., Silva, A. P. C., Fernandes, H. A., Almeida, S. P., \& Mendonça, H. (2015). Escala para avaliação de estressores psicossociais no contexto laboral: construção e evidências de validade. Psicologia: Reflexão e Crítica, 28(2), 340-349. Doi:10.1590/16787153.201528214.

Ferreira, M. L. C. B., \& Siqueira, M. M. M. (2005). Antecedentes de intenção de rotatividade: estudo de um modelo psicossocial. Revista Organizações em Contexto-online, 1(2), 47-67.

Field, A. (2009). Descobrindo a estatística usando o SPSS-2. Porto Alegre, RS: Artmed.

Gil-Monte, P. R. (2005). El sindrome de quemarse por el trabajo ("burnout”). Una enfermedad laboral en la sociedad del bienestar. Madrid: Pirámide.

Gomide Jr., S. (1999). Antecedentes e consequentes das percepções de justiça no trabalho. Tese de doutorado, Universidade de Brasília, Brasília, Brasil.

Hom, P. W., \& Kinicki, A. J. (2001), Toward a Greater Understanding of How Dissatisfaction Drives Employee Turnover. Academy of Management Journal, 44, 975-987.

Huang, Y. H., Lee, J., McFadden, A. C., Murphy, L. A., Robertson, M. M., Cheung, J. H., \& Zohar, D. (2016). Beyond Safety Outcomes: an Investigation of the Impact of Safety Climate on Job Satisfaction, Employee
Engagement and Turnover Using Social Exchange Theory as the Theoretical Framework. Applied Ergonomics, 55, 248257. Doi: 10.1016/j.apergo.2015.10.007.

Jones, K. (2013). Weighing the Demand for Drivers. Commercial Carriers Journal, 1, 5460.

Jou, R. C., Kuo, C. W., \& Tang, M. L. (2013). A Study of Job Stress and Turnover Tendency among Air Traffic Controllers: The Mediating Effects of Job Satisfaction. Transportation research part E: Logistics and transportation review, 57, 95-104.

Krueger, G. P. (2013). Health and wellness for commercial truck, bus and motor coach drivers: Wellness report. Park Ridge, IL: American Society of Safety Engineers.

Lannoo, S., \& Verhofstadt, E. (2016). What Drives the Drivers? Predicting Turnover Intentions in the Belgian Bus and Coach Industry. Transportation Research Part A: Policy and Practice, 91, 251-259.

LeMay, S. A., Williams, Z., \& Garver, M. (2009). A Triadic View of Truck Driver Satisfaction. Journal of Transportation Management, 20(2), 19-36.

Lings, S., \& Leboeuf-Yde, C. (2000). WholeBody Vibration and Low Back Pain: A Systematic, Critical Review of the Epidemiological Literature 1992-1999. International Archives of Occupational and Environmental Health, 73(5), 290-297. 
Macedo, I. (2008). Como o clima psicológico e o bemestar afectivo no trabalho explicam as intenções de abandono das organizações. Dissertação de mestrado, Instituto Superior de Ciências do Trabalho e da Empresa, Aveiro, Portugal.

Marôco J. (2007). Análise estatística com utilização do SPSS (3a ed.). Lisboa: Edições Sílabo.

Maroco, J., \& Garcia-Marques, T. (2006). Qual a fiabilidade do alfa de Cronbach? Questões antigas e soluções modernas?. Laboratório de Psicologia, 4(1), 65-90.

Marqueze, E. C., Ulhôa, M. A., \& Moreno, C. R. (2012). Irregular Working Times and Metabolic Disorders among Truck Drivers: A Review. Work, 41(1), 37183725. Doi:10.3233/WOR-2012-00853718.

Masson, V. A., \& Monteiro, M. I. (2010). Estilo de vida, aspectos de saúde e trabalho de motoristas de caminhão. Revista Brasileira de Enfermagem, 63(4), 533-540. Doi:10.1590/S0034-71672010000400006.

Min, H., \& Lambert, T. (2002). Truck Driver Shortage Revisited. Transportation Journal, 42(2), 5-17.

Mobley, W. H. (1977). Intermediate Linkages in the Relationship between Job Satisfaction and Employee Turnover. Journal of Applied Psychology, 62(2), 237-240.

Mobley, W. H. (1982). Employee Turnover: Causes, Consequences and Control. London: Addison-Wesley.
Moreno, C. R. D. C., \& Rotenberg, L. (2009). Fatores determinantes da atividade dos motoristas de caminhão e repercussões à saúde: um olhar a partir da análise coletiva do trabalho. Revista Brasileira de Saúde Ocupacional, 34(120), 128-138. Doi:10.1590/s0303-76572009000200004.

Movimento União Brasil Caminhoneiro (2017). Falta de caminhoneiros no país. Recuperado de

http://uniaobrasilcaminhoneiro.org.br/in dex.htm.

Nadler, S. S., \& Kros, J. F. (2014). Logistic Regression Approach to Predicting Truck Driver Turnover. International Journal of Applied Logistics (IJAL), 5(1), 15-32.

Narciso, F. V., \& Mello, M. T. D. (2017). Segurança e saúde dos motoristas profissionais que trafegam nas rodovias do Brasil. Revista de Saúde Pública, 5(26), 17. Doi:10.1590/S15188787.2017051006761 .

Oliveira, D. B. (2012). A reestruturação produtiva e o trabalho dos motoristas de caminhão: um estudo de caso em duas empresas de transporte rodoviário de carga. Dissertação de mestrado, Universidade de São Carlos, São Carlos, São Paulo, Brasil.

Pinto, C. A. A. (2012). Interface trabalho-familia em motoristas profissionais. Dissertação de mestrado, Universidade do Minho, Braga, Portugal.

Quinlan, M., \& Wright, L. (2008). Remuneration and Safety in the Australian Heavy Vehicle 
Industry: A Review Undertaken for the

National Transport Commission. Melbourne, Austrália: Project Report.

Resende, P. T. V., Souza, P. R., \& Cerqueira, P. R. (2010). Hábitos de vida e segurança dos caminhoneiros brasileiros. In Simpósio de Administração da Produção, Logística e Operações Internacionais. Anais do SIMPOI 2010. São Paulo, Brasil, $1-17$.

Ribeiro, F. H. (2008). Análise da percepşão das condições de trabalho, ambiente e saúde dos motoristas em Rio Verde - GO. Dissertação de mestrado, Universidade Católica de Goiás, Goiânia, Goiás, Brasil.

Schonfeld, I. S. (1996). Relation of Negative Affectivity to Self-Reports of Job Stressors and Psychological Outcomes. Journal of Occupational Health Psychology, 1, 397-412. Doi:10.1037/1076-8998.1.4.397.

Seshagiri, B. (1998). Occupational Noise Exposure of Operators of Heavy Trucks. American Industrial Hygiene Association, 59(3), 205-213.

Shattell, M., Apostolopoulos, Y., Sonmez, S., \& Griffin, M. (2010). Occupational Stressors and the Mental Health of Truckers. Issues in Mental Health Nursing, 31(9), 561-568. Doi:10.3109/01612840.2010.488783.

Silva, A. R., \& Silva, I. S. (2015). Conflito trabalho-família: um estudo com motoristas profissionais. Revista Psicologia Organizações e Trabalho, 15(4), 419-430. Doi: org/10.17652/rpot/2015.4.432
Silva, L. G. D., Luz, A. A. D., Vasconcelos, S. P., Marqueze, E. C., \& Moreno, C. R. D. C. (2016). Vínculos empregatícios, condições de trabalho e saúde entre motoristas de caminhão. Revista Psicologia Organizações e Trabalho, 16(2), 153-165. Doi:10.17652/rpot/2016.2.675.

Silva, R. A. (2015). Vida de caminhoneiro: sofrimento e paixão. Dissertação de mestrado, Pontifícia Universidade Católica, Campinas, São Paulo, Brasil.

Simmonds, E. G. S. (2015). Fatores determinantes da qualidade de vida no trabalho de motoristas de transporte de cargas em trânsito pela região de Barra do Garças - MT. In Congresso Internacional de Administração, Ponta Grossa, PR, Brasil.

Siqueira, M. M. M. (2001). Comprometimento organizacional afetivo, calculativo e normativo: evidências acerca da validade discriminante de três medidas brasileiras. Anais do Enanpad, 25, 23-40.

Staplin, L., \& Gish, K. W. (2005). Job Change Rate as a Crash Predictor for Interstate Truck Drivers. Accident Analysis \& Prevention, 37(6), 1035-1039.

Suzuki, Y. (2007). Truck Driver Turnover: What Rate Is Good Enough? International Journal of Physical Distribution \& Logistics Management, 37(8), 612-630. Doi:10.1108/09600030710825685.

Suzuki, Y., Crum, M. R., \& Pautsch, G. R. (2009). Predicting Truck Driver Turnover. Transportation Research Part E: 
Logistics and Transportation Review, 45(4), 538-550.

Swartz, S. M., Douglas, M. A., Roberts, M. D., \& Overstreet, R. E. (2017). Leavin'on my mind: influence of safety climate on truck drivers' job attitudes and intentions to leave. Transportation Journal, 56(2), 184209.

Tolentino, N. (2012). Histórias de vida de caminhoneiros: quem serão os caminhoneiros de amanhã?. São Paulo: Edicon.

Ulhôa, M. A., Marqueze, E. C., Lemos, L. C., Silva, L. G. D., Silva, A. A., Nehme, P., \& Moreno, C. R. D. C. (2010). Distúrbios psíquicos menores e condições de trabalho em motoristas de caminhão. Revista de Saúde Pública, 44(6), 1130-1136. Doi:10.1590/S0034-89102010000600019.

Van der Beek, A. J. (2012). World at Work: Truck Drivers. Occupational and Environmental Medicine, 69(4), 291-295. Doi:10.1136/oemed-2011-100342.

Zhang, Y., \& Feng, X. (2011). The Relationship between Job Satisfaction, Burnout, and Turnover Intention among Physicians from Urban State-Owned Medical Institutions in Hubei, China: A CrossSectional Study. BMC health services research, $11(1), 235$.

Williams Jr., D. F., Thomas, S. P., \& Liao-Troth, S. (2017). The Truck Driver Experience: Identifying Psychological Stressors from the Voice of the Driver. Transportation
Journal, 56(1), 54-76.

Doi:10.5325/transportationj.56.1.0054.

Recebido em: 5/1/2018

Aprovado em: 26/5/2018 